\title{
Systematic Computations on Amicable Numbers
}

\section{By J. Alanen, O. Ore and J. Stemple}

The first pair of amicable numbers beyond the classical $(220 ; 284)$ was obtained by Fermat in 1636 . Since this time a considerable number of amicable pairs

$$
(M ; N), \quad M<N,
$$

have been discovered. The most recent lists are due to Escott [1], Poulet [2] and Garcia [3]. The paper by Escott contains the 390 pairs known by 1946 together with the names of their discoverers. The list of 43 pairs computed by Poulet was communicated to N. G. W. Beeger in 1941, but published only in 1948 after Poulet's death. The paper by Garcia contains 160 new pairs obtained by him and his students.

These pairs have all been found by examining numbers of special forms. By combining such results Dickson [4] was able to show that up to $M \leqq 6232$ there are only five amicable pairs:

$$
(220 ; 284) \quad(1184 ; 1210) \quad(2620 ; 2924) \quad(5020 ; 5564) \quad(6232 ; 6368) .
$$

The present calculations have been performed systematically, testing each number up to $M<10^{6}$. The work was done on the Yale IBM 7094 computer. The results are given in the following table; the computations also produce the perfect numbers $<10^{6}$. Altogether there are 42 pairs of amicable numbers below $10^{6}$. Among these there are 9 new ones, not previously listed; these have been marked by an asterisk. The calculations required two hours computer time.

$$
\begin{aligned}
& \text { Pairs of amicable numbers } \\
& 220=2^{2} \cdot 5 \cdot 11 \\
& 1184=2^{5} \cdot 37 \\
& 2620=2^{2} \cdot 5 \cdot 131 \\
& 5020=2^{2} \cdot 5 \cdot 251 \\
& 6232=2^{3} \cdot 19 \cdot 41 \\
& 10744=2^{3} \cdot 17 \cdot 79 \\
& 284=2^{2} \cdot 71 \\
& 1210=2 \cdot 5 \cdot 11^{2} \\
& 2924=2^{2} \cdot 17 \cdot 43 \\
& 5564=2^{2} \cdot 13 \cdot 107 \\
& 6368=2^{5} \cdot 199 \\
& 12285=3^{3} \cdot 5 \cdot 7 \cdot 13 \\
& 10856=2^{3} \cdot 23 \cdot 59 \\
& 17296=2^{4} \cdot 23 \cdot 47 \\
& 14595=3 \cdot 5 \cdot 7 \cdot 139 \\
& 63020=2^{2} \cdot 5 \cdot 23 \cdot 137 \\
& 18416=2^{4} \cdot 1151 \\
& 66928=2^{4} \cdot 47 \cdot 89 \\
& 76084=2^{2} \cdot 23 \cdot 827 \\
& 67095=3^{3} \cdot 5 \cdot 7 \cdot 71 \\
& 66992=2^{4} \cdot 53 \cdot 79 \\
& 69615=3^{2} \cdot 5 \cdot 7 \cdot 13 \cdot 17 \\
& 71145=3^{3} \cdot 5 \cdot 17 \cdot 31 \\
& { }^{*} 79750=2 \cdot 5^{3} \cdot 11 \cdot 29 \\
& 100485=3^{2} \cdot 5 \cdot 7 \cdot 11 \cdot 29 \\
& 122265=3^{2} \cdot 5 \cdot 11 \cdot 13 \cdot 19 \\
& 87633=3^{2} \cdot 7 \cdot 13 \cdot 107 \\
& 88730=2 \cdot 5 \cdot 19 \cdot 467 \\
& 124155=3^{2} \cdot 5 \cdot 31 \cdot 89 \\
& 122368=2^{9} \cdot 239 \\
& 141664=2^{5} \cdot 19 \cdot 233 \\
& 142310=2 \cdot 5 \cdot 7 \cdot 19 \cdot 107 \\
& 139815=3^{2} \cdot 5 \cdot 13 \cdot 239 \\
& 123152=2^{4} \cdot 43 \cdot 179 \\
& 153176=2^{3} \cdot 41 \cdot 467 \\
& 168730=2 \cdot 5 \cdot 47 \cdot 359
\end{aligned}
$$

Received May 31, 1966. 


\begin{tabular}{|c|c|}
\hline $171856=2^{4} \cdot 23 \cdot 467$ & $176336=2^{4} \cdot 103 \cdot 107$ \\
\hline $176272=2^{4} \cdot 23 \cdot 479$ & $180848=2^{4} \cdot 89 \cdot 127$ \\
\hline${ }^{*} 185368=2^{3} \cdot 17 \cdot 29 \cdot 47$ & $203432=2^{3} \cdot 59 \cdot 431$ \\
\hline $196724=2^{2} \cdot 11 \cdot 17 \cdot 263$ & $202444=2^{2} \cdot 11 \cdot 43 \cdot 107$ \\
\hline${ }^{*} 280540=2^{2} \cdot 5 \cdot 13^{2} \cdot 83$ & $365084=2^{2} \cdot 107 \cdot 853$ \\
\hline $308620=2^{2} \cdot 5 \cdot 13 \cdot 1187$ & $389924=2^{2} \cdot 43 \cdot 2267$ \\
\hline${ }^{*} 319550=2 \cdot 5^{2} \cdot 7 \cdot 11 \cdot 83$ & $430402=2 \cdot 7 \cdot 71 \cdot 433$ \\
\hline $356408=2^{3} \cdot 13 \cdot 23 \cdot 149$ & $399592=2^{3} \cdot 199 \cdot 251$ \\
\hline $437456=2^{4} \cdot 19 \cdot 1439$ & $455344=2^{4} \cdot 149 \cdot 191$ \\
\hline${ }^{*} 469028=2^{2} \cdot 7^{2} \cdot 2393$ & $486178=2 \cdot 7^{2} \cdot 11^{2} \cdot 41$ \\
\hline $503056=2^{4} \cdot 23 \cdot 1367$ & $514736=2^{4} \cdot 53 \cdot 607$ \\
\hline $522405=3^{2} \cdot 5 \cdot 13 \cdot 19 \cdot 47$ & $525915=3^{2} \cdot 5 \cdot 13 \cdot 29 \cdot 31$ \\
\hline $600392=2^{3} \cdot 13 \cdot 23 \cdot 251$ & $669688=2^{3} \cdot 97 \cdot 863$ \\
\hline $609928=2^{3} \cdot 11 \cdot 29 \cdot 239$ & $686072=2^{3} \cdot 191 \cdot 449$ \\
\hline $624184=2^{3} \cdot 11 \cdot 41 \cdot 173$ & $691256=2^{3} \cdot 71 \cdot 1217$ \\
\hline $635624=2^{3} \cdot 11 \cdot 31 \cdot 233$ & $712216=2^{3} \cdot 127 \cdot 701$ \\
\hline $643336=2^{3} \cdot 29 \cdot 47 \cdot 59$ & $652664=2^{3} \cdot 17 \cdot 4799$ \\
\hline${ }^{*} 667964=2^{2} \cdot 11 \cdot 17 \cdot 19 \cdot 47$ & $783556=2^{2} \cdot 31 \cdot 71 \cdot 89$ \\
\hline $726104=2^{3} \cdot 17 \cdot 19 \cdot 281$ & $796696=2^{3} \cdot 53 \cdot 1879$ \\
\hline${ }^{*} 802725=3 \cdot 5^{2} \cdot 7 \cdot 11 \cdot 139$ & $863835=3 \cdot 5 \cdot 7 \cdot 19 \cdot 433$ \\
\hline${ }^{*} 879712=2^{5} \cdot 37 \cdot 743$ & $901424=2^{4} \cdot 53 \cdot 1063$ \\
\hline $898216=2^{3} \cdot 11 \cdot 59 \cdot 173$ & $980984=2^{3} \cdot 47 \cdot 2609$ \\
\hline $947835=3^{3} \cdot 5 \cdot 7 \cdot 17 \cdot 59$ & $1125765=3^{3} \cdot 5 \cdot 31 \cdot 269$ \\
\hline${ }^{*} 998104=2^{3} \cdot 17 \cdot 41 \cdot 179$ & $1043096=2^{3} \cdot 23 \cdot 5669$ \\
\hline
\end{tabular}

Procedure. A pair of numbers (1) is amicable when

$$
\sigma(M)=\sigma(N)=M+N
$$

where $\sigma(M)$ denotes the sum of all divisors of $M$. When one introduces

$$
\sigma_{0}(M)=\sigma(M)-M
$$

the condition (2) changes into

$$
\sigma_{0}(M)=N, \quad \sigma_{0}(N)=M .
$$

This also leads to

$$
\sigma_{0}\left(\sigma_{0}(M)\right)=\sigma_{0}{ }^{(2)}(M)=M .
$$

In the calculations the straightforward summation of the divisors of each $M$ to obtain $\sigma_{0}(M)$ to check (5) was considered. However, a time estimate showed that this would require about 20 hours of computer time.

Consequently it was decided to base the computations upon the prime factorisation of $M$. When $N=\prod p_{i}^{\alpha_{i}}$ then

$$
\sigma_{0}(N)=\frac{\amalg I p_{i}^{\alpha_{i}+1}-1}{p_{i}-1}-N .
$$

(See for instance Ore [5, p. 89].) When

$$
\sigma_{0}(M)>M
$$


then $\sigma_{0}(M)$ was also factorized and a check for (5) was made.

Observation 1. Perfect numbers are defined by the relation

$$
\sigma_{0}(M)=M
$$

while amicable numbers $\left(M_{0}, \sigma_{0}(M)\right)$ satisfy $(5)$. This makes it natural to study in general the numbers $M$ satisfying a periodicity relation,

$$
\sigma_{0}^{(n)}(M)=M, \quad n=3 .
$$

Our calculations show that for $n=3$ no such number $M<10^{6}$ exists.

Such periodicity questions have been studied particularly by Catalan, Dickson and Poulet. (For references see Dickson [6, Chapter 1] and Lehmer [7, pp. 5-6].) Poulet [8] succeeded in finding the relations

$$
\sigma_{0}^{(5)}(12496)=12496, \quad \sigma_{0}{ }^{(28)}(14316)=14316 .
$$

Observation 2. It is not known that there exists an infinite number of amicable pairs. However, if this is the case the lists of amicable numbers suggest that one may have

$$
\lim M / N \rightarrow 1, \quad M \rightarrow \infty .
$$

An extension of the preceding table would throw further light upon such a conjecture. In support one may cite the series of amicable pairs defined by the ancient formula of Thabit ben Korrah. (See Ore [5, pp. 98-99].) If they represent an infinite set of amicable numbers they must satisfy (8).

Observation 3. Our table shows that both numbers in an amicable pair are in most cases even, although in some cases both are odd. In no case is one even, the other odd. The conjecture that there are no even-odd amicable pairs has been tested to a much higher limit than that used in our table. This check is based upon the following considerations:

When one of the numbers $M$ and $N$ in (2) is odd, the other even, their sum $\sigma(M)$ is odd. When $p$ is an odd prime the factor

$$
1+p+\cdots+p^{\alpha}=\left(p^{\alpha+1}-1\right) /(p-1)
$$

in (6) is odd only when $\alpha$ is even, that is

$$
M=2^{\alpha_{0}} \cdot m^{2}, \quad N=2^{\beta_{0}} \cdot n^{2}
$$

where $m$ and $n$ are odd. Thus one of the numbers $M$ and $N$ is an odd square, for instance, $M=m^{2}$. The relation (5) has been checked for such odd numbers $m$. The calculations are greatly facilitated by the fact that $M$ and $m$ have the same prime factors. One finds that for

$$
M \leqq 3469563409
$$

there is no odd square number $M$ which is one of a pair of amicable numbers. The bound given above had to be selected for the reason that no more computer time was available. An extension of our conjecture would be that there are no amicable pairs in which one number is a square.

Yale University

New Haven, Connecticut 
1. E. B. Escotr, "Amicable numbers," Scripta Math., v. 12, 1946, pp. 61-72. MR 8, 135. p. 77.

2. P. Poulet, "Forty-three new couples of amicable numbers," Scripta Math., v. 14, 1948,

3. M. Garcia, "New amicable pairs," Scripta Math., v. 23, 1957, pp. 167-171. MR 20 *5158.

4. L. E. Dickson, "Theorems and tables on the sum of the divisors of a number," Quart. J. Pure Appl. Math., v. 44, 1913, pp. 264-296.

5. O. ORE, Number Theory and Its History, McGraw-IIill, New York, 1948. MR 10, 100.

6. L. E. Dickson, History of the Theory of Numbers, Carnegie Institution Publ. no. 256, 1919; reprint, G. E. Stechert, 1934.

7. D. H. Lenmer, Guide to Tables in the Theory of Numbers, Bull. Nat. Research Council, no. 105, National Research Council, Washington, D. C., 1941. MR 2, 247.

8. P. Poulet, La Chasse aux Nombres, Vol. 1, Stevens, Brussels, 1929.

\title{
Primes of the Form $n^{4}+1$
}

\author{
By M. Lal
}

In this note we report 172 new primes of the form $n^{4}+1$ and tabulate all such primes for $1 \leqq n \leqq 4004$.

Factorization of the numbers of the form $n^{4}+1$ has been extensively studied by Cunningham [1] and Gloden [2], [3]. They used a sieve method based on the four solutions of the congruence equation

$$
x^{4}+1 \equiv 0 \quad(\bmod p)
$$

for all primes of the form $8 k+1$. With primes less than $4 \times 10^{6}$, numbers $n^{4}+1$ for $n \leqq 2000$ have been completely factorized.

For $p>4 \times 10^{6}$, it becomes rather difficult and time consuming to solve (1). Consequently, it renders such a sieve less practical. An analysis of the growing inefficiency of such a sieve with increasing $p$ is given in $[5, \mathrm{p}$. 188]. However, the range for $n$ can be extended by using Alway's method [4] of factorization modified for odd divisors of the form $8 k+1$ and testing each number $n^{4}+1$ individually.

With the modified Alway's method, we found all primes for $1 \leqq n \leqq 1000$. This was done to check the program and to provide independent data which is not readily accessible for this interval. The search was then extended to $2000 \leqq n \leqq$ 4004; 172 primes and one prime factor for other composite numbers were indentified. The time required to establish the primality of $n=4002$ is 2.0 hours on the IBM 1620 computer Model II. As the project required several hundred hours of machine time, the search was made on three IBM 1620 computers-one at Memorial and two at Kingston. All primes of the form $n^{4}+1$, complemented by those given in [3] for $1000<n<2040$, are presented in Table 1 .

Discussion of Results. Shanks [5] has made a conjecture regarding the number of primes $Q(N)$ of the form $n^{4}+1$ for $1 \leqq n \leqq N$ and has given the following expression:

$$
Q(N) \sim .66974 \int_{2}^{N} \frac{d n}{\log n} .
$$

The observed count and those computed by using (2), rounded to the first decimal, are given below in Table 2. 